\title{
OPTIMAL CONTROL ON AN INFINITE DOMAIN
}

\author{
B. D. CRAVEN ${ }^{1}$
}

(Received 13 June, 2003; revised 24 February, 2005)

\begin{abstract}
For an optimal control problem with an infinite time horizon, assuming various terminal state conditions (or none), terminal conditions for the costate are obtained when the state and costate tend to limits with a suitable convergence rate. Under similar hypotheses, the sensitivity of the optimum to small perturbations is analysed, and in particular the stability of the optimum when the infinite horizon is truncated to a large finite horizon. An infinite horizon version of Pontryagin's principle is also obtained. The results apply to various economic models.
\end{abstract}

\section{Introduction}

Various economic models lead to optimal control problems with an infinite time horizon, which go beyond the standard optimal control theory for a finite time interval $[0, T]$. There are unresolved questions for infinite horizon concerning convergence, boundary conditions as time tends to infinity, and stability of an optimum to small perturbations. Some results are obtained, for a class of problems that can be converted to standard control problems by a nonlinear transformation of the time variable.

For an optimal control problem, whose objective is an integral over a time domain $[0, T]$, subject to a differential equation for the state function $x(\cdot)$ in terms of the state and the control $u(\cdot)$, and constraints on the control, necessary conditions for an optimum (assuming some regularity conditions) consist of:

- the differential equation for the state, with an initial condition;

- the differential equation for the costate, with a terminal condition;

- the Pontryagin maximum (or minimum) principle.

These necessary conditions become also sufficient for an optimum, if the functions defining the problem also possess suitable convex, or generalised convex, properties.

\footnotetext{
${ }^{1}$ Department of Mathematics and Statistics, University of Melbourne, VIC 3010, Australia; e-mail:craven@ms.unimelb.edu.au.
}

(C) Australian Mathematical Society 2005, Serial-fee code 1446-1811/05 
The terminal condition for the costate is often called a transversality condition.

In some applications, especially in economics, optimal control problems arise, with an infinite domain $[0, \infty)$ replacing $[0, T]$. (See for example Leonard and Long [5], and Chiang [1].) Then the proofs of the above necessary conditions only partly apply. If an optimum over $[0, \infty)$ is assumed, with state $x^{*}(\cdot)$, then necessary conditions may be deduced, by considering an optimum over $[0, T]$ with an adjoined terminal condition $x(T)=x^{*}(T)$. This approach does not give the terminal condition for the costate, but it is needed for any discussion of sufficient conditions. For infinite domain and no terminal condition for the state, some conditions are known when the costate tends to zero as time tends to infinity. Michel [6] considered a nonnegative objective integrand, and assumed that the optimal state was contained in a suitable neighbourhood of feasible solutions. Janin [4] assumed a coercivity restriction on the dynamic equation, and a discount rate not too small. But questions remain when the terminal state is constrained.

An optimal control problem may be expressed as optimising a function $J(x, u)$ of state and control, subject to a differential equation $D x=M(x, u)$, mapping the functions $x$ and $u$ to the gradient $D x$, and to constraints on $u$. Here $x$ and $u$ must lie in appropriate spaces $X$ and $U$, such as piecewise smooth functions with norm $\|x\|_{\infty}+\|D x\|_{\infty}$, and piecewise continuous functions with norm $\|u\|_{\infty}$. (See Section 2 for a definition of piecewise smooth on an infinite domain.) Then necessary Lagrangian conditions for an optimum hold, involving a Lagrange multiplier $\theta$, attached to the constraint $-D x+M(x ; u)=0$. If the time domain is $[0, T]$, then the element $\theta$ in the dual space of $X$ can be represented by $\varphi \xi=\int_{0}^{T} \lambda(t) \xi(t) d t$ for each $\xi \in X$, where $\lambda$ is a generalised function, which reduces in the present context to an ordinary function, sometimes with delta functions added. Then the differential equation for the costate $\lambda(\cdot)$, together with the terminal condition on $\lambda(T)$, follow from the Lagrangian necessary conditions.

But not all dual spaces (consisting of continuous linear functions on $X$ ) can be thus represented by a function $\lambda(t)$. In particular, this representation fails when the time domain is $[0, \infty)$. In an economic context, $\lambda(t)$ has the significance of a price or a unit cost. But this interpretation is not always available over an infinite domain; qualitatively, something additional may happen "at infinity".

However, if the state and control functions are assumed to tend to finite limits (with values not necessarily specified) as $t \rightarrow \infty$, then a nonlinear transformation of time $t \in[0, \infty)$ to $\tau \in[0,1]$, with $\tau=1$ included in the domain because the limits exist, converts the optimal control problem on $[0, \infty)$ to an optimal control problem of standard form on $[0,1]$. Some further assumption on the rate of convergence is needed, in order that required continuity and boundedness properties are satisfied. Then the boundary condition for the costate can be established. These conditions are specified in Theorems 2.1 and 2.2 in Section 2 below. Theorem 3.1 gives the boundary 
condition.

This transformation of the time scale also allows the sensitivity of the optimal point to a small perturbation to be analysed for an infinite time domain, by considering an equivalent problem on $[0,1]$. A known theorem about the stability of a strict minimum to a small perturbation can then be applied. The results are given in Section 3. A version of the Pontryagin principle for an infinite time domain is given in Section 4.

Convergence rate assumptions are implicit, though not always explicit (see Chiang [1]), in some standard economic models over infinite time. Thus, following Ramsey [7], a modified objective $\int_{0}^{\infty}(f(x(t), u(t))-b) d t$ may be considered, with no discount factor but a limiting value $b$ subtracted from the integrand. But this objective is only finite if the control (and the state, if present in the integrand) tend to limits sufficiently fast.

Applications of these results to economics are discussed in Craven and Islam [3, Chapter 9].

\section{A tractable class of control problems on an infinite domain}

Consider the optimal control problem

$$
\begin{aligned}
& \underset{x(\cdot), u(\cdot)}{\operatorname{minimise}} F(x, u):=\int_{0}^{\infty} e^{-\delta t} f(x(t), u(t)) d t \\
& \text { subject to } \begin{cases}x(0)=x_{0}, \quad \dot{x}(t)=m(x(t), u(t), t) & (t \geq 0), \\
u_{\text {low }} \leq u(t) \leq u_{\text {high }} & (t \geq 0) .\end{cases}
\end{aligned}
$$

Assume that $f(\cdot, \cdot)$ and $m(\cdot, \cdot, \cdot)$ are differentiable. Assume that $u(\cdot)$ is piecewise continuous, $x(\cdot)$ is piecewise smooth, and additionally that the limits

$$
x(\infty):=\lim _{t \rightarrow \infty} x(t) \text { and } u(\infty):=\lim _{t \rightarrow \infty} u(t)
$$

exist, with values not necessarily specified. It follows that $\dot{x}(t) \rightarrow 0$ as $t \rightarrow \infty$. (Here $x$ is defined as piecewise smooth on $[0, \infty)$ when the transformed function $\hat{x}$ on $[0,1]$, as defined below, is piecewise smooth.) The rate of convergence is not determined. However, if it is fast enough (say exponential), then the following hypothesis becomes plausible.

HYPOTHESIS CR. For some function $\psi(\cdot) \geq 0$ with $\int_{0}^{\infty} \psi(t) d t$ finite, the weighted function $\psi(\cdot)^{-1} m(x, u(\cdot), \cdot)$ is bounded, and differentiable, uniformly in $t$, with the first derivatives $\psi(\cdot)^{-1} m_{x}(x(\cdot), u(\cdot), \cdot)$ and $\psi(\cdot)^{-1} m_{u}(\dot{x}(\cdot), u(\cdot), \cdot)$ also bounded, with $(x, u)$ in a neighbourhood of the optimal $(\bar{x}, \bar{u})$. 
In order to obtain the differential equation for the costate, the functions $J$ and $M$ must be shown to be differentiable (where the mapping $M$ is defined by

$$
M(x, u)(t):=m(x(t), u(t), t))
$$

and the Lagrange multiplier must be representable by a costate function on $[0, \infty)$. The adjoint equation will follow from

$$
F_{x}(\bar{x}, \bar{u})-\theta\left(D-M_{x}(\bar{x}, \bar{u})\right)=0,
$$

which is part of the necessary Karush-Kuhn-Tucker conditions for an optimum $(\bar{x}, \bar{u})$ of the control problem, with a Lagrange multiplier $\theta$, and which hold (see for example Craven [2]) equally for infinite as for finite time domains. Consider then a nonlinear time transformation $t=\omega(\tau)$, with $\omega(\cdot)$ increasing concave, $\omega(0)=0$, and $\omega(\tau) \rightarrow \infty$ as $\tau \rightarrow 1$. An example is

$$
t=\omega(\tau)=\frac{1}{2} \zeta \ln \left(\frac{1+\tau}{1-\tau}\right), \quad \tau=\tanh \left(\frac{\omega}{\zeta}\right), \quad \omega^{\prime}(\tau)=\frac{\zeta}{1-\tau^{2}} .
$$

Define the transformed functions $\hat{x}(\tau)=x(t)$ and $\hat{u}(\tau)=u(t)$. A costate function $\hat{\lambda}(\tau)$ will be found, and then the costate $\lambda(t):=\hat{\lambda}(\tau)$. The functions $u$ and $x$ are given the norms stated in Section 1 , on $[0, \infty)$; correspondingly $\hat{u}$ and $\hat{x}$ are given these norms on $[0,1]$. For brevity, write $x(\infty):=\lim _{t \rightarrow \infty} x(t), u(\infty):=\lim _{t \rightarrow \infty} u(\infty)$, $\lambda(\infty):=\lim _{t \rightarrow \infty} \lambda(t)$ (when the last limit is shown to exist). In general, $x(t)$ has components $x^{i}(t)$, with corresponding components $\lambda^{i}(t)$.

THEOREM 2.1. Assume that the control problem reaches an optimum on the infinite time domain $[0, \infty)$, with the discount term $e^{-\delta t}$ in the objective, and that Hypotheses (KKTx) and $\mathrm{CR}$ hold. Then the mappings $F$ and $M$ are Fréchet differentiable, and the adjoint differential equation holds:

$$
-\dot{\lambda}(t)=e^{-\delta t} f_{x}(\bar{x}(t), \bar{u}(t))+\lambda(t) m_{x}(\bar{x}(t), \bar{u}(t), t) \quad(0 \leq t<\infty),
$$

with the boundary condition

$\lambda^{i}(\infty)=0$ when $x^{i}(\infty)$ is free, $\quad \lambda^{i}(\infty)$ is free when $x^{i}(\infty)$ is prescribed

Proof. From CR, giving $x$ and $u$ the norms stated in Section 1,

$$
\begin{aligned}
(M(x, u)-M(\bar{x}, \bar{u}))(t)= & m(x(t), u(t), t)-m(\bar{x}(t), \bar{u}(t), t)) \\
= & \left.m_{x}(\bar{x}(t), \bar{u}(t), t)\right)(x(t)-\bar{x}(t)) \\
& \left.+m_{u}(\bar{x}(t), \bar{u}(t), t)\right)(u(t)-\bar{u}(t))+r(t),
\end{aligned}
$$

in which $\left.\left.m_{x}(\bar{x}(t), \bar{u}(t), t)\right)(x(t)-\bar{x}(t))=O(\psi(t)), m_{u}(\bar{x}(t), \bar{u}(t), t)\right)(u(t)-\bar{u}(t))=$ $O(\psi(t)),|r(t)|<\epsilon O(\psi(t))$ when $\|x-\bar{x}\|<\delta(\epsilon)$ and $\|u-\bar{u}\|<\delta(\epsilon)$. 
This shows that $M$ is Fréchet differentiable, with

$$
\left.M_{x}(\bar{x}, \bar{u})(x-\bar{x})=\int_{0}^{\infty} m_{x}(\bar{x}(t), \bar{u}(t), t)\right)(x(t)-\bar{x}(t)) d t
$$

bounded by const $\int_{0}^{\infty} \psi(t)\|x-\bar{x}\|_{\infty} d t$; and a similar result for $M_{u}$.

The differentiability of $J$ is proved similarly, with the discount term $e^{-\delta t}$ fulfilling the role of $\psi(t)$ for $M$. In order to represent the multiplier $\theta$ in $(K K T x)$ by a function $\lambda(\cdot)$, transform the given control problem on $[0, \infty)$ to an equivalent control problem on $[0,1]$ by the nonlinear time transformation $t=\omega(\tau)$ given above. The equivalent problem is

$$
\begin{aligned}
& \underset{\hat{x}(\cdot), \hat{u}(\cdot)}{\operatorname{minimise}} \int_{0}^{1}\left(\frac{1+\tau}{1-\tau}\right)^{-\zeta \delta / 2} f(\hat{x}(\cdot), \hat{u}(\cdot), \omega(\tau)) \frac{\zeta}{1-\tau^{2}} d \tau \\
& \text { subject to }\left\{\begin{array}{l}
\hat{x}(0)=x_{0}, \quad \frac{d \hat{x}(\tau)}{d \tau}=m(\hat{x}(\cdot), \hat{u}(\cdot), \omega(\tau)) \frac{\zeta}{1-\tau^{2}}, \\
u_{\text {low }} \leq \hat{u}(\tau) \leq u_{\text {high }}
\end{array} \quad(t \geq 0) .\right.
\end{aligned}
$$

Note that $\tau=1$ is included in the domain, because the limits $\hat{x}(1)=x(\infty)$ and $\hat{u}(1)=u(\infty)$ exist, given $\mathrm{CR} ; \hat{x}$ is piecewise smooth and $\hat{u}$ is piecewise continuous on the closed interval $[0,1]$; and $\omega^{\prime}(\tau)=\zeta\left(1-\tau^{2}\right)^{-1}$.

Since the derivatives $F_{x}$ and $M_{x}$ also exist, the differential equation for the costate $\lambda(\cdot)$ can be deduced, in the usual way. For each function $\hat{v} \in C^{1}([0,1]), D \hat{v}(\tau)=$ $\hat{v}^{\prime}(\tau) / \omega^{\prime}(\tau)$, so integration by parts gives:

$$
\begin{aligned}
-\int_{0}^{1} \hat{\lambda}(\tau)(D \hat{v}(\tau)) \omega^{\prime}(\tau) d \tau & =-\int_{0}^{1} \hat{\lambda}(\tau) \hat{v}^{\prime}(\tau) d \tau \\
& =-[\hat{\lambda}(\hat{\tau}) \hat{v}(\tau)]_{0}^{1}+\int_{0}^{1} \hat{\lambda}^{\prime}(\tau) \hat{v}(\tau) d \tau \\
& =-[\hat{\lambda}(\tau) \hat{v}(\tau)]_{0}^{1}+\int_{0}^{1}(D \lambda(\tau)) \omega^{\prime}(\tau) \hat{v}(\tau) d \tau .
\end{aligned}
$$

Then substitution for the Fréchet derivatives in $(K K T x)$ gives

$$
\begin{aligned}
0 & =\int_{0}^{1}\left\{\left(\frac{1+\tau}{1-\tau}\right)^{-\zeta \tau / 2} f_{x}+\hat{\lambda}(\tau)\left(-D+m_{x}\right)\right\} \hat{v}(\tau) \omega^{\prime}(\tau) d \tau \\
& =\int_{0}^{1}\left\{\left(\frac{1+\tau}{1-\tau}\right)^{-\zeta \tau / 2} f_{x}+\hat{\lambda}(\tau) m_{x}+D \hat{\lambda}(\tau)\right\} \hat{v}(\tau) \omega^{\prime}(\tau) d \tau+[\hat{\lambda}(\tau) \hat{v}(\tau)]_{0}^{1}
\end{aligned}
$$

and after integrating by parts

$$
=\int_{0}^{\infty}\left\{e^{-\delta t} f_{x}+\lambda(t) m_{x}+D \lambda(t)\right\} v(t) d t+[\lambda(t) v(t)]_{0}^{\infty}
$$


after transforming from $\tau$ to $t$. In the first two lines, $f_{x}$ and $m_{x}$ have $\hat{x}(\tau)$ and $\hat{u}(\tau)$ as arguments; in the third line they have $x(t)$ and $u(t) ; \hat{\lambda}(\tau)=\lambda(t)$; and $\hat{v}(\tau)=v(t)$. Then $\{\ldots\}=0$, giving the stated costate differential equation, if the integrated part vanishes. Considering $\hat{v}(\cdot)$ as an increment to $x(\cdot), v(0)=0$; and $v(\infty)=\hat{v}(1)=0$ in the case when $x(\infty)$ is specified. So the integrated part vanishes, as required, when $\lambda_{i}(\infty):=0$ provided $x_{i}(\infty)$ is unspecified.

Suppose now that a terminal constraint $r(x(\infty) \geq 0$ is adjoined to the optimal control problem. For the transformed problem with $\tau \in[0,1]$, this becomes $r(\hat{x}(1)) \geq 0$. (This constraint may be assumed active, so optimal $r(\hat{x}(1))=0$, since inactive components may be omitted.) This constraint may be equivalently replaced (in a neighbourhood of the optimum) by a penalty term

$$
\frac{1}{2} \mu\left\|\left[-r(\hat{x}(\tau))+\mu^{-1} \sigma\right]_{+}\right\|^{2} \delta(\tau-1),
$$

where $\sigma$ is a nonnegative Lagrange multiplier, determined by the constraint, and $\delta(\cdot)$ is Dirac's delta-function. This puts a term $\sigma r^{\prime}(\hat{x}(\tau)) \delta(\tau-1)$ on the right side of the adjoint differential equation. Integrating over the delta-function (see Craven [2]) gives a terminal condition $\hat{\lambda}(1)=\sigma r^{\prime}(\hat{x}(1))$; and $\lambda(\infty)$ has the same value. This has proved the following theorem.

THEOREM 2.2. For the control problem on an infinite time domain, with terminal state condition $r\left(\lim _{t \rightarrow \infty} x(t)\right) \geq 0$, under the hypotheses of Theorem 2.1 , the bound ary condition for the costate is $\lim _{t \rightarrow \infty} \lambda(t)=\sigma r^{\prime}\left(\lim _{t \rightarrow \infty} x(t)\right)$, with the nonnegative multiplier $\sigma$ determined by the terminal state condition.

REMARK. If the discount term is omitted from the objective function, but instead Hypothesis CR holds for the integrand $f$ as well as for $m$, then the conclusion of Theorems 2.1 and 2.2 still hold. This includes the Ramsey case mentioned in Section 1 , writing now $f(x(\cdot), u(\cdot))$ for $f(x(\cdot), u(\cdot))-b$.

\section{Perturbing the control problem}

A perturbation theory is available for the mathematical program

$$
P(q): \underset{z}{\operatorname{minimise}} F(z, q) \text { subject to }-G(z, q) \in S,
$$

in which $q$ is a perturbation parameter (with datum value 0 ), $X, Y, U$ are normed spaces, the functions $F: X \times U \rightarrow \mathbb{R}$ and $G: X \times U \rightarrow Z$ are differentiable, and $S$ is a closed convex cone. The following basic perturbation result was given by 
Craven [2, Lemma 7.4.7]; here, assumption (c) is weakened to what the proof actually assumes. A strict minimum at $z=a$ (when $q=0$ ) means that

$$
F(z, 0)-F(a, 0) \geq \rho(r)>0 \quad \text { whenever } \quad\|z-a\|=r \quad \text { and } \quad G(z, 0) \in S,
$$

for some function $\rho(\cdot)>0$ of positive $r$, sufficiently small.

THEOREM 3.1 (Perturbation of a strict minimum). Assume that:

(a) $P(0)$ reaches a strict local minimum at $z=a$;

(b) $F(\cdot, \cdot)$ and $G(\cdot, \cdot)$ are uniformly continuous, when $q$ is in a bounded neighbourhood $N$ of 0 in $U$, and $x$ is in a bounded neighbourhood of the feasible set $E(q)$ for $P(q)$, with $q$ in $N$;

(c) for each nonzero $q \in U, E(q)$ is nonempty, and $F(\cdot, q)$ reaches a minimum on $E(q) \cap B(a, r)$, for each sufficiently small $r>0$.

Then, if $\|q\|$ is sufficiently small, $P(q)$ reaches a local minimum at $z^{*}(q)$, where $z^{*}(q) \rightarrow 0$ as $\|q\| \rightarrow 0$.

The uniform continuity (b) is immediate for compact neighbourhoods in finite dimensions, but is nontrivial for infinite dimensions. Likewise (c) needs additional proof for infinite dimensions, except in the case (see [2]) where $P(q)$, for nonzero $q$, happens to be a finite-dimensional problem. For infinite dimensions, assumption (c) assumes the attainment of a minimum at $z^{*}(q)$, when $q$ is nonzero; and then the theorem proves that $z^{*}(\cdot)$ is continuous at 0 , showing that the perturbation is stable.

This theory will be applied first to an optimal control problem:

$$
V(q):=\underset{u}{\operatorname{minimise}} J(u, q),
$$

where

$$
\begin{aligned}
J(u, q) & :=\int_{0}^{T} f(x(t), u(t), t, q) d t+R(x(T), q) \\
& =\int_{0}^{T}[f(x(t), u(t), t, q)+\delta(x-T) R(x(T), q)] d t
\end{aligned}
$$

subject to

$$
x(0)=x_{0}, \quad \dot{x}(t)=m(x(t), u(t), t, q), \quad u(t) \in E(t) \quad(0 \leq t \leq T) .
$$

The objective can be written as $J(u, q)$, since the differential equation determines $x(\cdot)$ in terms of $u(\cdot)$, say as $x(\cdot)=\Phi(u(\cdot), q)$. There may be a terminal condition on $x(T)$; if so, it is included in $R(x(T))$.

PROPOSITION 3.2. For the unperturbed problem $(q=0)$, and finite horizon $T$, assume that $f(\cdot, \cdot, t, 0), m(\cdot, \cdot t, 0)$, and $R(.$.$) have bounded first partial derivatives.$ 
Denote $x=\Phi(u)$ and $\hat{x}=\Phi(\hat{u})$, and assume the norms $\|u\|_{1}$ (or $\|u\|_{\infty}$ and $\|x\|_{\infty}+$ $\left.\|D x\|_{\infty}\right)$. Then the following Lipschitz properties hold:

$$
\begin{aligned}
& \left(\exists \kappa_{1}\right)(\forall u, \hat{u} \in \mathbf{D}) \quad\|\Phi(u)-\Phi(u)\| \leq \kappa_{1}\|u-\hat{u}\| ; \\
& (\forall u, \hat{u} \in \mathbf{D}) \quad\|J(u)-J(\hat{u})\| \leq \kappa_{2}\|u-\hat{u}\| .
\end{aligned}
$$

PROOF. (Lip state): Let $w:=u-\hat{u}$ and $y:=x-\hat{x}$. Then

$$
|y(t)| \leq \int_{0}^{t}[|a(s)||y(s)|+|b(s)||w(s)|] a d s \equiv \int_{0}^{t}|a(s)||y(s)| d s+v(t),
$$

where $a(s)$ and $b(s)$ are bounded above by suprema of partial derivatives of $m$ over suitable neighbourhoods. From Gronwall's inequality,

where

$$
|y(t)| \leq v(t)+\int_{0}^{t} \pi(t, s) v(s) d s,
$$

$$
\pi(t, s)=\exp \left(\int_{s}^{t}|a(r)| d r\right)|a(s)| .
$$

Now $v(t) \leq\left\|\left.b\right|_{\infty}\right\| w \|_{1}$, with norms taken over $[0, T]$, noting that the norm $\|w\|_{1}$ is appropriate for the Pontryagin theory. If $\mu:=\|a\|_{\infty}$, then $\pi(t, s) \leq \mu \exp (\mu(t-s))$. Hence

$$
|y(t)| \leq v(t)+e^{\mu t}\|v\|_{1} \leq v(t)+e^{\mu t}\|b\|_{\infty}\|w\|_{1} \leq \text { const }\|w\|_{1}
$$

for $T<\infty$. Moreover, from the differential equation for $\dot{x}(t)$,

$$
\|D x-D \hat{x}\|_{\infty} \leq\left\|m_{x}\right\|_{1}\|y\|_{\infty}+\left\|m_{u}\right\|_{\infty}\|w\|_{1} \leq \text { const }\|w\|_{1},
$$

or

$$
\|D x-D \hat{x}\|_{\infty} \leq\left\|m_{x}\right\|_{1}\|y\|_{\infty}+\left\|m_{u}\right\|_{1}\|w\|_{\infty} \leq \text { const }\|w\|_{\infty} .
$$

(Lip obj): If $A, B$ and $C$ are upper bounds of the first partial derivatives of $f$ and $R$, then

$$
\begin{aligned}
|J(u)-J(\hat{u})| & \leq \int_{0}^{T}[A|y(t)|+B|w(t)|+\delta(t-T) C|y(t)| d t, \\
& \leq\left(B+\kappa_{\mathrm{l}}(A+C)\right)\|w\| .
\end{aligned}
$$

The results follow.

Now consider the perturbation parameter $q \neq 0$. Assume that the bounds given by $\|a\|,\|b\|, A, B$ and $C$ now hold whenever $|q|<\gamma$ for some $\gamma>0$. Then (Lip state) 
and (Lip obj) hold uniformly in $q$. Thus hypothesis (b) of the perturbation theorem is verified. (Since $\|u\|_{1} \leq T\|u\|_{\infty}$, the norm $\|u\|_{\infty}$ could also be used here.) For a control problem with $T$ replaced by $\infty$ and $R$ omitted, assume an optimum is reached at $(\bar{x}, \bar{u})$ when $q=0$. An appropriate hypothesis is $\mathrm{CRq}$, namely that $\mathrm{CR}$ holds when $f$ and $m$ depend also on the parameter $q$, uniformly when $(x, u)$ are in a neighbourhood of the optimal $(\bar{x}, \bar{u})$ and $|q|<\gamma$, for some $\gamma>0$.

PROPOSITION 3.3. Assume that the infinite-time problem satisfies Hypothesis CRq. Then (Lip state) and (Lip obj) hold, for the norms $\|u\|_{\infty}$ and $\|x\|_{\infty}+\|D x\|_{\infty}$.

PROOF. The proof of Proposition 3.2 is modified as follows. In the proof of (Lip state), $a(r)=O(\psi(r))$, hence $\pi(t, s)=O(\psi(s)), b(s)=O(\psi(s))$, so that $v(t) \leq$ const $\|w\|_{\infty}$; then $|y(t)| \leq v(t)+$ const $\|w\|_{1}$; so $\|y\|_{\infty} \leq$ const $\|w\|_{\infty}$. A similar modification applies to the proof of (Lip obj), replacing $A, B$ and $C$ by $O\left(e^{-\delta t}\right)$; then, uniformly in $|q|<\gamma,|J(u)-J(\hat{u})| \leq$ const $\|u-\hat{u}\|_{\infty}$.

THEOREM 3.4. For the control problem with parameter $q$, over an infinite time interval $[0, \infty)$, assume Hypothesis $\mathrm{CRq}$, and assume that the problem reaches a strict minimum when $q=0$, and reaches a minimum for $0 \neq|q|<\gamma$ subject to bounds on $|u(t)|$. Then the control problem with parameter $q$ reaches a local minimum at $(x, u)=\left(x_{q}^{*}, u_{q}^{*}\right)$, when $|q|$ is sufficiently small, where $\left(x_{q}^{*}, u_{q}^{*}\right) \rightarrow(\bar{x}, \bar{u})$ as $q \rightarrow 0$.

PROOF. In Theorem 3.1, hypothesis (a) holds by assumption, at a point $(\bar{x}, \bar{u})$; hypothesis (b) holds by Proposition 3.3, given CRq; and hypothesis (c) holds by assumption. Hence, by Theorem 2.2, the result holds.

REMARK. This theorem assumes existence of a minimum, and then proves stability, so that the perturbed optimal state will be close to the original optimal state.

Now consider the approximation of the control problem on $[0, \infty)$ by a problem on $[0, T]$, with a suitably large horizon $T$. Equivalently, the reformulated problem with nonlinear time $\tau \in[0,1]$ replaces $f$ and $m$ by zero for $\tau \in(1-q, 1]$, where $T=\omega(1-q)$. Here, $q$ may be considered as a perturbation parameter.

THEOREM 3.5. Assume that the optimal control problem with infinite time interval $[0, \infty)$, with $x(\infty)$ unconstrained, reaches a strict minimum $(\bar{x}, \bar{u})$, and assume Hypothesis $\mathrm{CR}$. Assume that the truncated problem, replacing $[0, \infty)$ by $[0, T]$, where $T$ is sufficiently large, reaches a minimum at $\left(\bar{x}_{T}, \bar{u}_{T}\right)$, subject to bounds on $|u(t)|$. Then $\left(\bar{x}_{T}, \bar{u}_{T}\right) \rightarrow(\bar{x}, \bar{u})$ as $T \rightarrow \infty$. 
Proof. In view of Proposition 3.3, it suffices to establish hypothesis (b) of Theorem 2.2 when $q$ is sufficiently small, for $1-q=\psi(T)$. Consider

$$
1-q \leq 1-q^{\prime}<1-q^{\prime \prime}<1 .
$$

Then the difference in the perturbed values of $M$, corresponding to $q^{\prime}$ and $q^{\prime \prime}$, is bounded by

$$
\text { const } \int_{1-q^{\prime}}^{1-q^{\prime \prime}} \omega(\tau) d \tau \stackrel{q^{\prime} \cdot q \rightarrow 0}{\longrightarrow} 0 .
$$

This proves the uniform continuity of $M$ in $q$. The same argument applies also to $M_{x}$ and $M_{u}$, and also to $F, F_{x}$, and $F_{u}$, here replacing $\psi(t)$ by $\exp (-\delta t)$.

\section{Pontryagin principle for an infinite domain}

The adjoint differential equation, with its boundary condition, was obtained in Theorem 2.1 for a control problem on an infinite time domain, by using a nonlinear time transformation. The Pontryagin minimum (or maximum) principle may be similarly approached. In the proof in Craven [2] for a finite horizon $T$, the hypotheses were that:

(i) the differential equation for $\dot{x}(t)$ is solvable for state $x(\cdot)$ as a Lipschitz function of control $u(\cdot)$;

(ii) $F$ and $M$ are partially differentiable with respect to $x$, uniformly with respect to $u$ near $\bar{u}$;

(iii) the constraints on the control $u$ have the form $(\forall t) u(t) \in \Gamma(t)$.

Here, hypothesis (ii) is fulfilled if $f(\cdot, \cdot)$ and $m(\cdot, \cdot, \cdot)$ have bounded second partial derivatives. This suffices to make an integral expression for

$$
F(x, u)-F(\bar{x}, u)-F_{x}(\bar{x}, \bar{u})(x-\bar{x})
$$

small when $\|x-\bar{x}\|$ is small, uniformly in $u$ near $\bar{u}$. But being bounded could be replaced by dominance by an integrable function.

This approach to the Pontryagin principle extends to a problem with infinite $T$, when reformulated on $[0,1]$ by a nonlinear time transformation, provided that $\mathrm{Hy}$ pothesis CR holds, and that also the second partial derivatives of $f(\cdot, \cdot)$ and $m(\cdot, \cdot, \cdot)$ are dominated by a function $\psi(\cdot) \geq 0$ for which $\int_{0}^{\infty} \psi(t) d t<\infty$.

\section{Acknowledgement}

The author thanks an anonymous referee for very careful checking of details, and a number of corrections. 


\section{References}

[1] A. C. Chiang, Elements of dynamic optimization (Waveland Press, Prospect Heights, Illinois, 2000).

[2] B. D. Craven, Control and optimization (Chapman and Hall, London, 1995).

[3] B. D. Craven and S. M. N. Islam, Optimization in economics and finance: some advances in non-linear, dynamic, multi-criteria and stochastic models (Springer, Amsterdam, 2005).

[4] R. Janin, "Conditions nécessaires d'optimalité dans un problème d'optimisation en horizon infini", C. R. Acad. Sci. Paris 289A (1979) 651-653.

[5] D. Leonard and N. V. Long, Optimal control theory and static optimization in economics (Cambridge University Press, Cambridge, UK, 1992).

[6] P. Michel, "On the transversality conditions in infinite horizon optimal problems", Econometrica 50 (1982) 975-985.

[7] F. Ramsey, “A mathematical theory of saving”, Economic J. 38 (1928) 543-559. 